\title{
A Zone-Based Hierarchical Framework and Clustering Scheme for Energy-Efficient Wireless Sensor Networks
}

\author{
Muhammad Zahid Khan, Madjid Merabti, Bob Askwith, Faycal Bouhafs \\ School of Computing and Mathematical Sciences, Liverpool John Moores University, UK \\ M.Zahid-Khan@2008.ljmu.ac.uk, \{M.Merabti, R.J.Askwith, F.Bouhafs\}@1jmu.ac.uk
}

\begin{abstract}
In Wireless Sensor Networks (WSNs), sensor-nodes are usually deployed with limited energy reserves in remote environments for a long period of time with less or no human intervention. It makes energy-efficiency as a challenging issue both for the design and deployment of WSNs. A novel aspect of our conducted research is the development of a new Zone-Based Hierarchical Framework (ZBHF) with integrated newly proposed Zone-Based Self-organizing Clustering (ZBSC) scheme for energy-efficient WSNS. The key feature of the proposed solutions is the minimization of energy consumption during the self-organization clustering process to maximize network lifetime. The scheme has been implemented and evaluated in a custom-designed Java based simulator. In the light of the conducted experiments proposed schemes have evolved as a better solution in addressing identified issues in comparison with LEACH, LEACH-C and BCDCP. Furthermore, it consumed less energy in sending sensed data to the base-station with an increased network lifetime.
\end{abstract}

Keywords - WSN; self-organization; clustering; ZBHF; ZBSC

\section{Introduction}

Wireless Sensor Networks (WSNs), can be considered as a special breed of wireless ad-hoc networks with reduced or zero mobility. These networks combine wireless communication (i.e. transceiver) and minimal on-board computation facilities (i.e. processor or micro-controller) with sensing and monitoring. All these components together in a single device constitute a so-called 'sensor node' or simply a Sensor. Sensors in a WSN, have limited energy reserves, which are unlikely to be recharged or replaced. Therefore, energyefficiency is one of the most critical issues in the design and deployment of WSNs, since it has a direct impact on the overall network lifetime. Techniques that utilize energyreserves efficiently in the operations of WSNs, can keep sensor-nodes operational for a relatively long period of time without any human intervention [1]. For example, sensornodes can use a considerable amount of energy simply to organize the deployed sensor-nodes into groups or clusters, which degrades network performance and network lifetime.

To prolong the operational lifetime of WSN, energyefficiency must be considered in almost every aspect of network design. In essence, network self-organization is one of the most important phases that utilize a considerable amount of sensor-node's energy. In this context, an energy-efficient network self-organization protocol or algorithm can provide significant power savings in individual sensor-node and thus extend the operational lifetime of an entire sensor network [2, $3]$.

According to Collier and Taylor [4], self-organization is the process of autonomous formation of connectivity, addressing and routing structure. A self-organized wireless node can be grouped or clustered into an easily manageable network infrastructure [5]. Every cluster has a leader node, commonly referred as the Cluster-Head $\mathrm{CH}$ ), which manages and organizes activities in the cluster. Grouping sensor-nodes in clusters to self-organizing the network has been widely investigated by the research community in order to achieve energy-efficiency in WSNs. Apart from supporting energyefficiency, clustering offer numerous advantages such as, optimized network management, efficient data aggregation and fault-tolerance, etc. [6].

The contribution of this work is to present a novel ZoneBased Hierarchical Framework (ZBHF) and Zone-Based Selforganization Clustering (ZBSC) scheme to conserve energy and maximize sensor network lifetime. The newly proposed ZBHF presents a novel network deployment infrastructure where the network is divided into $n$-zones, and each zone is deployed with a resource-rich heterogeneous node called a zone-manager (ZM). The deployed sensor-nodes have $n$-tier hierarchy, such that we have high-level manager nodes (such as $\mathrm{ZM}$ and $\mathrm{CH}$ nodes) and low-level managed nodes (such as common sensor-nodes). Network management tasks such as clustering, and data aggregation are shifted to high-level nodes in the hierarchy to reduce the energy-consumption of resourcescarce low-level sensor-nodes. Furthermore, using the ZBHF, as an underlying network deployment infrastructure, a new Zone-Based Self-organization Clustering (ZBSC) scheme is proposed. ZBSC self-organizes the network into various clusters. The new ZBHF and ZBSC scheme reduces the energy cost of self-organization clustering, and minimizes the number of messages exchanged among sensor-nodes.

In our recent work [7], we have proposed a novel architecture for fault-tolerance and management of WSNs, but the new architecture did not provide the experimental results. Thus, this paper is a continuation of our earlier work, which provides further developments and refinements of the earlier 
proposed architecture. In ZBHF and ZBSC, we extend the earlier work in four ways: Firstly, we propose a method to divide the sensor network into $n$-zones; secondly, we build $n$ tier hierarchy of managed and management nodes and properly distribute the management task to different nodes based on their role in the network. Thirdly, we propose a new clustering scheme to self-organize sensor-nodes in an energy-efficient way. Fourthly, we evaluate our framework and clustering scheme through simulation and compare it with existing relevant research.

The rest of the paper is organized as follows. Section II summarizes the most relevant previous work. Section III and IV, describes the proposed ZBHF and ZBSC scheme in detail. Simulation results are presented in Section V. Conclusions and future work are given in Section VI.

\section{RELATED WORK}

A number of surveys on self-organization clustering algorithms and schemes can be found in $[6,8]$. We especially consider the class of the clustering algorithms, which organizes the sensor network into a set of clusters. In a typical clustering scenario, sensor-nodes are deployed over a sensorfield and a distributed cluster-head selection algorithm is executed to form clusters. In WSN, clustering is to used increase network capacity, maximize throughput, minimize network delay, and conserve energy and so on [9]. In the recent past the concept of decentralized hierarchical management with heterogeneous sensor-node is used excessively to bring energy-efficiency in WSNs. In a hierarchical architecture, high-energy nodes (e.g. $\mathrm{CH}$ ) can be used to aggregate and process information, while low-energy nodes (e.g. member sensor-nodes) can be used to perform sensing and forwarding. Some of the well-known hierarchical cluster-based protocols are: LEACH (Low-Energy Adaptive Clustering Hierarchy) and LEACH-C (Centralized) [10, 11], and BCDCP (Base-station Controlled Dynamic Clustering Protocol) [12].

LEACH [9, 10] is one of the most cited protocol, specifically designed to provide energy-efficiency and maximize network lifetime. The main objective of LEACH is to guarantee a certain network lifetime while minimizing energy consumption in the $\mathrm{CH}$ selection and cluster formation processes. Energy-efficiency is achieved by ensuring that all nodes die at the same time by rotating the role of $\mathrm{CH}$ periodically among the nodes within the cluster. However, $\mathrm{LEACH}$ performs a random selection of $\mathrm{CHs}$, where, there exists a probability that a node with low-energy is selected as a $\mathrm{CH}$. When this node dies, the whole cluster becomes dysfunctional. Furthermore, to form clusters messages are broadcasted over the network, which also drains excessive amount of energy. Finally, $\mathrm{CH}$ sends the aggregated data directly to the far away base-station, which further exhausts the battery of that node; therefore, it is unsuitable for a resource constrained WSNs.

LEACH-C (Centralized) [8] was proposed to address the weakness of LEACH protocol. LEACH-C uses a centralized approach for self-organization. The algorithm begins from the base-station where each node sends its location information and energy-level to base-station. CHs are selected randomly, but base-station ensures that a low-energy does not become a $\mathrm{CH}$. The main drawback of LEACH-C is that it is not feasible for large-scale networks because nodes, which are far away from the base-station, may have difficulty in sending information to it. Furthermore, it also consumes excessive energy in sending such information to a faraway base-station.

BCDCP [12] is another known scheme using the idea of Minimal Spanning Tree (MST) to connect CHs and randomly choose a leader node to send data to the sink. BCDCP makes the best use of a high-energy sink to choose $\mathrm{CHs}$ and form clusters by an iterative cluster splitting algorithm. It performs uniform placement of $\mathrm{CHs}$ throughout the whole sensory fields, and utilizes $\mathrm{CH}$-to- $\mathrm{CH}$ routing to transfer the data to the base station. BCDCP reduces energy dissipation of the network more than LEACH and LEACH-C; however, the iterative clustering splitting technique is more energy intensive when forming the clusters to self-organize the network. In addition, their network topology constrains them from being applied in a large-scale network.

Over the time researchers have also used new techniques for network self-organization such as biologically inspired or artificial intelligence based solutions [13, 14]. Such solution may provide effective solutions in specific cases, by only considering certain parameters and models; however, they suffer from high-energy consumption and limited applicability for resource-constrained large-scale WSNs. In the light of the above it could be concluded that there is still a need of new self-organizing solutions to address the problems associated with the energy-efficiency and network lifetime in WSNs.

\section{A ZONE BASED HIERARCHICAL FRAMEWORK}

It is a well-known fact that the energy-efficiency and lifetime of WSNs is highly influenced by self-organization and clustering process. More importantly, in cluster-based sensor networks, efficiency is further affected by the distance between sensor-nodes and base-station, and the amount of data aggregated and forwarded to the base-station. To address this important issue, we first propose a new Zone-Based Hierarchical Framework (ZBHF) followed by a new ZoneBased Self-Organization Clustering (ZBSC) scheme to selforganize the network into clusters in an energy-efficient way.

In the proposed ZBHF, initially we divide the sensing region or network into a number of zones. Each zone is considered separately and assigned a resource-rich heterogeneous node known as zone-manager. Each zone may be or may not be further divided into sub-zones. Further division of zone will depend upon the size of the sensing region and density of nodes in that zone. Then, a hierarchical management approach was employed among the deployed sensor-nodes to arrange them in a hierarchy of managed and management nodes (e.g. ZM as a high-level hierarchical node and $\mathrm{CH}$ as a low-level hierarchical node, etc.). This role assignment is based on the type of node and its resources. By 
adopting the ZBHF as a new network deployment infrastructure, we propose a new ZBSC scheme, which selforganizes all sensor-nodes into multiple clusters inside each zone. By using the ZBHF for self-organization clustering, the $\mathrm{ZM}$ nodes help to distribute and delegate the management tasks (e.g. $\mathrm{CH}$ selection, $\mathrm{CH}$ rotation, data aggregation, etc.) throughout the whole network which significantly reduces the message exchange between nodes and a base-station, hence conserve bandwidth and energy. It will allow all the sensornodes to carry out local data fusion and aggregation, and encourage to aggregate similar packets locally at the lowerlevel (e.g. at the $\mathrm{CH}$ ) to reduce the number of transmissions.

Finally, zone division help us to balance the distribution of cluster-heads in the network which ultimately avoid the concentration of cluster-heads in close vicinity, hence provide full coverage and connectivity. Furthermore, it guarantees that each node belongs to one and only one cluster, which provides full coverage of all the deployed sensors and support scalability as well.

\section{A. Network Model and Specifications}

In this section, we explain the underlying concept of the ZBHF, and describe the network model, specifications and assumptions. For the development of ZBHF, we made some assumptions about the deployed sensor-nodes and the underlying network model. A set of static and homogenous sensor-nodes $\mathrm{S} N_{s}$ are deployed with random distribution over an area $A$ of interest. For simplicity, we assume that the cluster is a circular region, and the $\mathrm{CH}$ is located at the centre of this region. Two nodes can communicate with each other directly if they are within the transmission range. Sensor-nodes are assumed to know their locations or relative position through location techniques such as recursive position estimation or virtual co-ordinate system [15].

\section{B. Zone-Formation in $\mathrm{ZBHF}$}

The two main key elements considered in the design of ZBHF are zone-formation and hierarchy of nodes, as illustrated in Fig 2, and 3. Network area can be divided into n-zone (four zones division shown by dotted lines in Fig. 1). A zone is a geographical division of the network area. For the illustration purposes we assume a square network area, initially divided into four zones by considering the network size. The number of zones is fixed at the initial setting; however, depending on the network size and node density, each zone can be further divided into sub-zones. Each zone is a subset of clusters, and each cluster is a subset of cluster-member nodes.

Zone-formation is carried out through the Cartesian Coordinate System (CCS), as shown in Fig. 1, i.e. the network field is divided into four zones; $\mathrm{Z} 1, \mathrm{Z} 2, \mathrm{Z} 3$, and $\mathrm{Z} 4$. We describe a sensor network to be in two-dimensional X, Y plane, written as $(\mathrm{X}, \mathrm{Y})$. To begin with, the Centre of the area $\mathrm{A}$ is calculated in a two dimensional $(\mathrm{X}, \mathrm{Y})$ plane. Then using the centre point as a reference point; mid-point on $\mathrm{X}$-coordinate and Y-coordinate is calculated, as stated in Equ.(1) and Equ.(2). The axes of two-dimensional Cartesian system divide the plane into four finite regions, called a quadrant, each bounded by two half-axes. Since $X$ and $Y$ are length and width respectively, therefore they are positive and consequently, the network plane area will be in the first quadrant. Let suppose $\mathrm{A}=200 \times 200 \mathrm{~m} 2$ is the area of the network, then according to CCS:

$\Rightarrow\left(X_{\min }, Y_{\min }\right)=(0,0), \quad\left(X_{\max }, Y_{\max }\right)=(200,200)$

To find out the center of the plane area we perform the following procedure:

$$
\begin{aligned}
& X_{\text {mid }}=\frac{X_{\text {min }}+X_{\text {max }}}{2} \\
& Y_{\text {mid }}=\frac{Y_{\text {min }}+Y_{\text {max }}}{2}
\end{aligned}
$$

Hence, $\left(X_{\text {mid }}, Y_{\text {mid }}\right)$ is the central point of the plane area. The area of each zone, as shown in Equ.(5) can be found by using Equ.(1) and Equ.(2), e.g. the area of Z1 is (see Fig. 1.):

$$
\begin{aligned}
& \Delta X=\left(X_{\text {mid }}-X_{\text {min }}\right) \\
& \Delta Y=\left(Y_{\text {mid }}-Y_{\text {min }}\right)
\end{aligned}
$$

Hence the area of a zone three $(\mathrm{Z} 1)$ is:

$$
A_{Z 1}=\Delta X \times \Delta Y
$$

i.e. from Equ.(5), the ares of zone-one can be calculated as: $A z l=100 \times 100 \mathrm{~m}^{2}$. As shown in Fig. 1, each sensor-node can calculate in which zone it currently resides based on its location information (i.e. its $X, Y$ co-ordinates) and using the following equation:

$Z M_{-} I D_{X}=\left(X_{-} X_{\text {min }}\right) / \Delta X$ and $Z M_{-} I D_{Y}=\left(Y-Y_{\text {min }}\right) / \Delta Y$

Where $Z M_{-} I D_{X}$ and $Z M_{-} I D_{Y}$ are the co-ordinate identifier $(X$, $Y$ ) of a zone-manager node, $X_{\min }$ and $Y_{\min }$ are the $X$ and $Y$ coordinates of the node with minimum co-ordinate in the network. The size of the zone $A_{Z}$ is calculated and made available to the sensors during initial network deployment. For simplicity, we have assigned different co-ordinates to each zones i.e. $Z 1=(0,0), Z 2=(0,1), Z 3=(1,1)$ and $Z 4=(1,0)$. By using the Equ.(6), sensor-nodes can calculate its location and it can find out to which zone it belongs to.

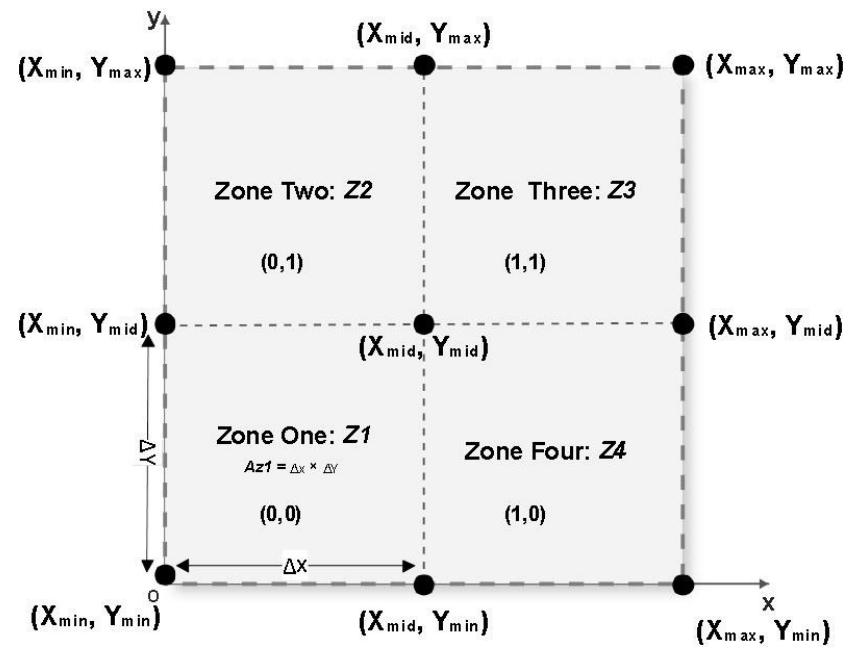

Figure 1. Zone-formation in ZBHF by CCS 
Once the zone-formation is completed, the central-manager (CM) or base-station is placed at the centre of the network field. Fig.3 presents the final layout of the network field division into four zones. A ZM node in each zone is placed at a location, so that it can cover all deployed sensor-nodes in its zone.

\section{Hierarchy of Nodes and Role Assignment}

To build the hierarchy and properly distribute management tasks in ZBHF, we propose $n$-tier hierarchy for WSNs. However, the number of hierarchical levels is based on application type, node density and number of zones. In the ZBHF we categorise participating sensor-nodes into different management roles: Sensor-Nodes (SN), Cluster-Head nodes $(\mathrm{CH})$, Zone-Manager nodes (ZM), and Central-Manager (CM) node. Roles for these nodes are assigned according to their resources (e.g. energy, processing, memory, etc.), responsibilities and hierarchy in the network. This hierarchical arrangement has various intrinsic advantages. For instance, sensor-nodes, which are normally unable to communicate due to limited radio signals, can be interconnected. Furthermore, the hierarchical structure manages large-scale WSNs efficiently by distributing the management load from a single entity to various managing nodes in the hierarchy. Role description of the participating nodes in the network is as follows (See Fig. 2):

- Central-Manager (CM) - The top-level node in the hierarchy, also known as a sink or base-station. It is responsible for the management of a whole network and takes the high-level management decisions (e.g. initiating a request for data aggregation, etc.).

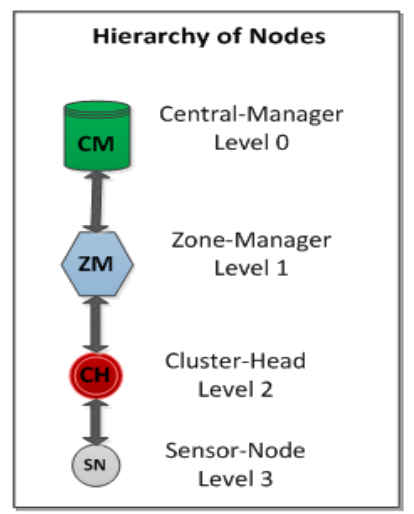

Fig. 2. Nodes Hierarchy

- Zone-Manager (ZM) - Resource-rich heterogeneous nodes, which reside at the second level of hierarchy in ZBHF. Each zone has one designated ZM node, which manages and coordinates the operations of all the associated lowerlevel nodes, (such as $\mathrm{CHs}$ ) in that zone.

- Cluster-Head $(\mathrm{CH})$ - These are the selected nodes from the ordinary homogenous sensor-nodes, and they reside at the third level of hierarchy. Each $\mathrm{CH}$ node has its associated Cluster-Member sensor-nodes, which communicate directly to their $\mathrm{CH}$.

- Sensor-Node (SN) - These are homogenous sensor-nodes, which are deployed over the field of interest to fully cover the monitoring area. This category represents the lowest level in the hierarchy. SNs perform the basic function of sensing and forwarding data to its associated $\mathrm{CH}$.

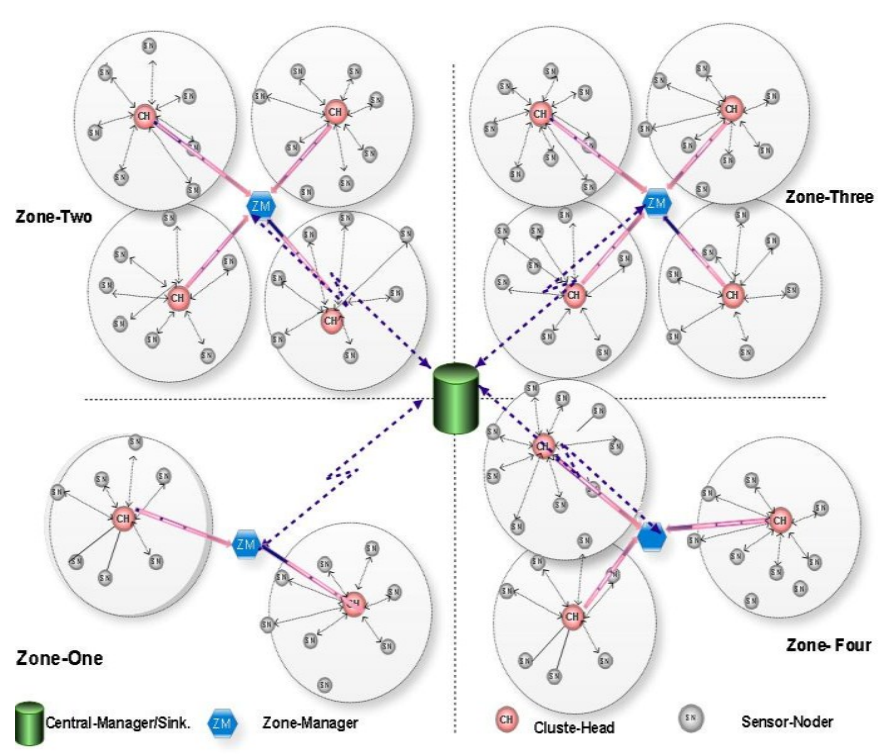

Fig.3. ZBHF: Network model and node deployment

ZBHF provides a novel WSN deployment infrastructure with well-organized model for energy-efficient network selforganization clustering and network management. In the next section, we present our new self-organization clustering scheme overlaid on ZBHF.

\section{A Zone-BAsed SElf-Organization Clustering Scheme}

The Zone-Based Self-Organization Clustering Scheme (ZBSC) self-organizes the network into various clusters in each zone. After self-organization, sensor-nodes are geographically grouped into clusters and capable of operating in two basic modes i.e. sensing mode and the $\mathrm{CH}$ mode. The novelty of our ZBSC scheme with respect to other clustering protocols is that it self-organizes the network with minimum energy consumption and maximize network lifetime. ZBSC algorithm consists of two phases, namely Network Initialization Phase and Cluster Configuration Phase.

\section{A. Network Initialization Phase}

Soon after the zone division and nodes deployment, networkinitialization process starts. The CM node broadcasts a Network Initialization Message (CM_NIM) to all one-hop ZM nodes. Each ZM node acknowledges the reception of the CM_NIM message and broadcasts it as a Zone Initialization Message (ZM_ZIM) to all the sensor-nodes within its radio range. Since the area of each zone has already been calculated in the zone-formation phase, therefore, the coverage radius of a $\mathrm{ZM}$ is tuned according to the size of a zone. A ZM_ZIM message contains the ZM Location (ZM_Loc) and the ZM identifier (ZM_ID). Sensor-nodes which are in the direct radio range of ZM that receive a ZM_ZIM, acknowledge it with by sending a Zone Initialization Acknowledgement Message (SN_ZIAM), which contain SN Location (SN_Loc), SN 
identifier (SN_ID), and SN Energy status (SN_Ec). In this way, every $\mathrm{SN}$ recognizes its zone and its associated $\mathrm{ZM}$ in the network. By receiving the SN ZIAM, the ZM will have complete information about all SNs in its zone. This information will help to elect a suitable $\mathrm{CH}$ node.

Message Filtering - To reduce the number of messages, we introduced a message filtering mechanism to avoid extra traffic generated in the network. Since every SN knows its correspondent ZM, therefore, if it receives a ZM ZIM message from another ZM it will simply discard it.

\section{B. Cluster Configuration Phase}

After the network initialization process, a cluster configuration phase starts in each zone. In this phase, a ZM first performs the key task of $\mathrm{CHs}$ selection, and when the $\mathrm{CHs}$ are selected, then SNs begins to group into clusters to complete the cluster configuration process. For initial deployment, ZM uses the SNs' location and current energy-level to select a $\mathrm{CH}$. The cluster configuration process is as follows:

- During the network initialization process, each ZM recognizes all $\mathrm{SNs}$ in its zone and builds a Zone Information Table (ZM_ITb). This table contains each SN identifier (SN_ID), its Location (SN_Loc), and current Energy status (SN_Ec). By analyzing the table, ZM selects those nodes as $\mathrm{CH}$ which has the highest energy-level.

- In the proposed ZBSC scheme, ZM selects 5\% of SNs as $\mathrm{CH}$ nodes in each zone. This is the optimum number of $\mathrm{CH}$ nodes in a given number of SNs in a network. The problem has been discussed in [10], and their results show that 5\% of nodes in the network operating as $\mathrm{CH}$ can achieve better performance with various network parameter settings. Using simulations, the authors found that the value 5\% works well for determining good clusters, and shows the overall decrease in the cost function and gives balance clusters. We use the same technique to select $5 \%$ nodes as $\mathrm{CH}$ nodes in each zone. This will give us the advantage to fully cover the network and balance energy load among nodes.

- Once the suitable and optimal CH nodes are selected; ZM transmits this information back to all SNs in its zone. This is done by broadcasting a message that contains the $\mathrm{CH}$ ID and ZM ID to each node. The receiving node matches its SN_ID with the CH_ID, if both the IDs matches, then that node take the role of a $\mathrm{CH}$; otherwise the node will become a cluster-member $\mathrm{SN}$. The selected $\mathrm{CHs}$ in each zone broadcast a $\mathrm{CH}$ Join Advertisement message ( $\mathrm{CH}$ JAdv) to every node in its radio range. This message contains the $\mathrm{CH}$ identifier and ZM identifier. Initially, each $\mathrm{SN}$ is in a nonassociated state. When a node receives a $\mathrm{CH}_{-} \mathrm{JAdv}$ message, it will evaluate the Received Signal Strength Indicator (RSSI) from each $\mathrm{CH}$ and choose the most suitable $\mathrm{CH}$ according to the signal strength of the advertisement message, and then acknowledges the message by sending a SN Join Request message (SN_JReq) to register to a chosen $\mathrm{CH}$ from which it receives highest signal strength. The SN JReq message contains SN ID, SN_Loc, and SN_Ec. The $\mathrm{CH}$ accepts the joining request, and a SN becomes a cluster-member SN of that cluster and connects with that $\mathrm{CH}$. Since each cluster-member node is in the communication range of a $\mathrm{CH}$, therefore, every node within the cluster transmits data directly data to its $\mathrm{CH}$ for aggregation. Figs. 4 demonstrate the cluster configuration process. Using this mechanism all SNs in each zone form cluster, and the network is self-organized into a fully connected WSN.

\section{ANALYSIS AND SIMULATION RESULTS}

In the light of conducted research, a custom-designed and purpose-build simulation framework has been used to carry out our evaluation experiments. It has been developed by our research group and utilized previously in similar research projects [16, 17]. The framework has been written in Java (JDK 1.7.0), because Java is platform independent and flexible in nature. The key factor behind the selection of this simulator lies in its capability of addressing WSN management related issues, more specifically self-organization and clustering. Furthermore, it provides a greater flexibility and focus on the development of algorithms and solutions for the particular sensor network scenario described above. For instance, it provides each sensor-node a simulated battery in order to measure the energy consumption. In addition, it supports scalable WSNs simulation with a functionality of adding new schemes easily.

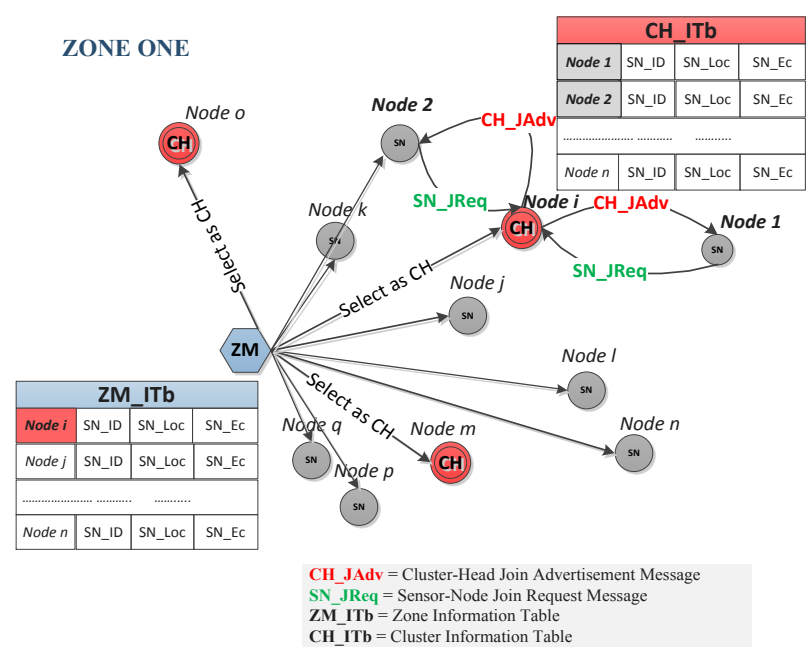

Fig.4. Cluster-configuration phase of ZBSC

We compared ZBSC scheme with other well-known selforganizing clustered schemes such as LEACH, LEACH-C and BCDCP. Performance is measured in terms of energy consumption during the self-organizing clustering process. For the sake of comparison, we use the same radio energy model and communication model used by LEACH, LEACH-C and BCDCP, namely, first-order order radio model $[9,11]$. Table I, shows our simulation parameters and network setup. Experiments were conducted to evaluate the performance of the proposed ZBSC scheme using clustering-cost metric because it is directly related to the sensor network selforganization. Clustering-cost refers to the total energy consumption during the cluster configuration phase, i.e. the sum of used energy of all the nodes in transmitting and 
receiving control messages during the cluster configuration process.

In the simulation, we generated different random setups, each with the above simulation settings. Assume that there are $M$ clusters, with $N / M$ sensor-nodes in each cluster on average, excluding the $\mathrm{CH}$. Thus, we will have $N / M-1$ sensor-nodes per cluster. Assume also that the size of the message is $K$, and $d$ is the distance ${ }^{1}$ between a node and the $\mathrm{CH}$. We use the same radio energy model to calculate the clustering-cost (energy) metric used in [10]. This metric implicitly captures the total operational energy cost of the transmission and receiving nodes in the network. The energy cost of receiving a bit is known to be smaller than the cost of sending a bit. If we annotate the energy consumed in transmitting $k$ bits of data with $E_{T x}$ Equ. (1) and the energy consumed for receiving with $E_{R x}$ Equ.(2):

TABLE I. SIMULATION PARAMETERS AND VALUES

\begin{tabular}{|l|l|}
\hline Parameters & Value \\
\hline Network Field $(A \times A)$ & $200 \times 200 \mathrm{~m}^{2}$ \\
\hline Number of Sensor-Nodes $(\mathrm{SNs})$ & 320 \\
\hline Number of Cluster-Heads $(\mathrm{CHs})$ & 0.05 \\
\hline Sensor-Nodes per Zone & $\approx 80$ \\
\hline Cluster-Heads per Zone & $\approx 4$ \\
\hline Sensor-Node Initial Energy (SN_Ei) & $2 \mathrm{~J}(2000 \mathrm{~mJ})$ \\
\hline Transmission Range of Sensor-Node & $50 \mathrm{~m}$ \\
\hline Transmission Range of Zone-Manager & $100 \mathrm{~m}$ \\
\hline Transmit Amplifier Energy $\left(\mathcal{E}_{\text {amp }}\right)$ & $100 \mathrm{pJ} / \mathrm{bit} / \mathrm{m}^{2}$ \\
\hline Radio Energy Dissipated $($ Eelec $)$ & $50 \mathrm{~nJ} / \mathrm{bit}$ \\
\hline Node Distribution & Random \\
\hline Packet Size $(\mathrm{K})$ & $48 \mathrm{bits}$ or 6 Bytes \\
\hline MAC Protocol & IEEE 802.11 \\
\hline
\end{tabular}

$$
\begin{aligned}
& E_{T x}(k, d)=\left(E_{\text {elec }} \times k\right)+\left(\varepsilon_{\text {amp }} \times k \times d^{2}\right) \\
& E_{R x}(k)=E_{\text {elec }} \times(k)
\end{aligned}
$$

We can calculate $d$, the Euclidean distance based on the location information by using the Pythagorean Theorem. In the equation below, $\mathrm{x}$ and $\mathrm{y}$ represent the location of the node, $a$ and $b$, on $\mathrm{x}$-axis and $\mathrm{y}$-axis.

$$
d_{a b}=\left[\left(a_{x}-b_{x}\right)^{2}+\left(a_{y}-b_{y}\right)^{2}\right]^{1 / 2}
$$

Thus, from Equ.(1) and Equ.(2), energy consumption of cluster-head (CH_Econs) and energy consumption of sensornode (SN_Econs) is given by:

$$
\begin{aligned}
& C H_{-} E \text { cons }=C_{-} E_{T x}+\sum_{i=0}^{\frac{N}{M}-1}\left(C H_{-} E_{R x}\right) \\
& S N_{-} E \text { cons }=\sum_{i=0}^{\frac{N}{M}-1}\left(S N_{-} E_{R x}+S N_{-} E_{T x}\right)
\end{aligned}
$$

Hence, total energy-consumption (Econsume) during the cluster configuration process is the sum of Equ. (3) and Equ. (4), which is in fact the energy spending in the message

\footnotetext{
${ }^{1}$ Gathering information about the communication channel between all nodes in impractical. Using distance is therefore an approximation of the amount of energy that will be required for communication.
}

exchange between $\mathrm{CH}$ and SNs. Therefore, the total energy consumption or clustering-cost is:

$$
\text { Econsume }=C_{-} E_{\text {cons }}+S N_{-} E_{\text {cons }}
$$

During the evaluation we have defined a Packet class. Each instance of the packet class contains data and its destination address. The packet size can be different for different applications according to the nature of the sensed data. For example, in an environmental monitoring application where sensor-nodes need to sense the temperature, a 1 byte of data may be enough. In our simulation we have evaluated various cases to calculate clustering-cost, as detailed below:

Case 1: In this case, we evaluate the clustering-cost of a single round during the cluster-configuration phase. We consider a group of $20 \mathrm{SNs}$, which includes one $\mathrm{CH}$ node. In ZBSC scheme, $\mathrm{ZM}$ and current $\mathrm{SNs}$ are involved in the $\mathrm{CH}$ selection process and cluster configuration process. To make an appropriate comparison, we have considered the most relevant schemes, i.e. LEACH, LEACH-C and BCDCP. Fig. 5 shows the energy-consumption (clustering-cost) in forming a cluster of $20 \mathrm{SNs}$ for each clustering algorithm. In the graph, LEACH and LEACH-C showed the highest level of energyconsumption, while BCDCP and ZBSC resulted in a small difference to each other. $\mathrm{LEACH}$ is simple in principle, but because it does not have location information about the sensornodes, which produces inefficient routing, and therefore higher energy-consumption. Furthermore, it sends an excessive number of control messages, which also consume great amount of sensor-node energy.

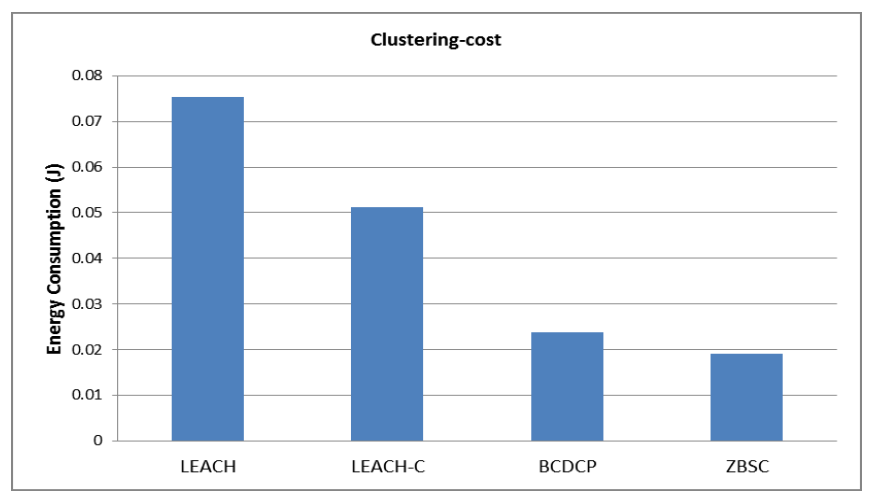

Fig.5. Case 1, Clustering-cost of forming a cluster

Both LEACH-C and BCDCP exhibit a reduction in energy consumption of 31 and 68 percent respectively over LEACH respectively, while in ZBSC the energy consumption is comparatively less. This is because all sensor-nodes in LEACH-C and BCDCP transmit messages to the distant basestation, which in turn causes significant energy loss in the nodes. However, in ZBSC no long-haul communication takes place which as a result conserves energy.

Case 2: In this case, we evaluate the energy-consumption of the self-organization clustering process per zone basis. In the simulation setup we consider $80 \mathrm{SNs}$ and $4 \mathrm{CHs}$ nodes per 
zone respectively. Fig. 6 shows the experimental results of the amount of energy consumed in first zone. It is the energy consumed during the formation of four clusters in the network topology of 80 nodes per zone. As expected, it was found that both LEACH and LEACH-C have a higher level of energyconsumption than the other two clustering algorithms. Overall, ZBSC has a lower energy-consumption. Fig. 7 shows the average energy dissipation over the number of various rounds (where one round is single cluster-configuration process) to self-organize the network into clusters. We run the simulation for 16 rounds, because we have $16 \mathrm{CHs}$ across the network.

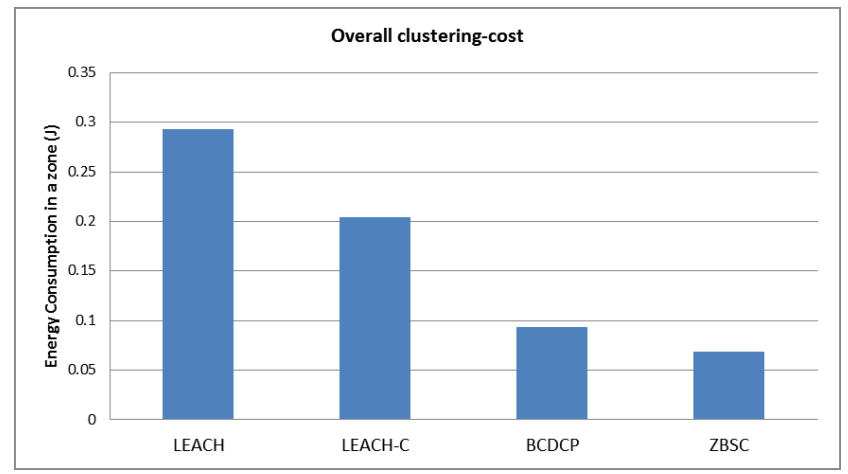

Fig. 6. Case 2, Clustering-cost of ZBSC

Fig. 7 shows the generation of 1 to 16 clusters in a network topology of 320 sensor-nodes for each clustering algorithm. It also shows the clustering-cost measurement during 16 rounds of cluster-configuration based on the number of each cluster generated. The results testify that ZBSC has significantly low clustering-cost as compared to $\mathrm{LEACH}$, LEACH-C and BCDCP; because ZBSC uses an alternative method that initially selects $\mathrm{CHs}$ based on its location and energy-level.

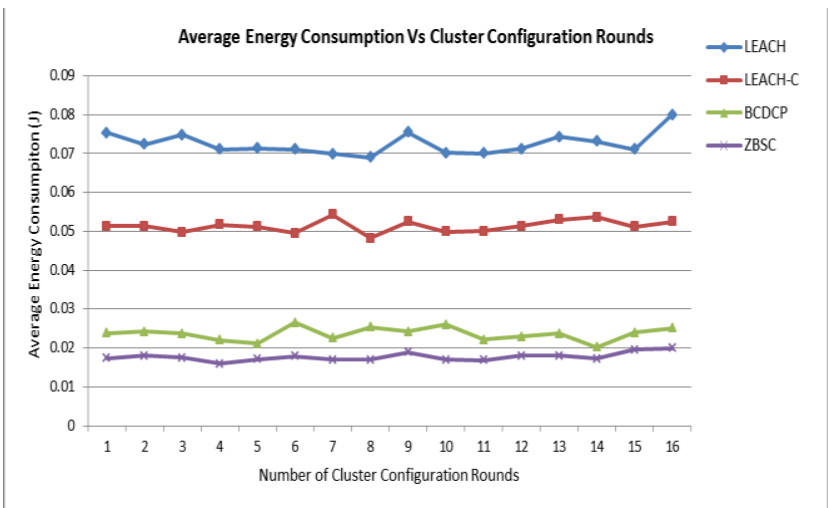

Fig.7. Case 2, Average energy-consumption in 16 rounds of clusterconfiguration

Case 3: In this case, we evaluate the amount of energy consume by sensor-nodes for sending the sensing data to the base-station in ZBHF. Several data gathering cycles can run, depending upon the nature of the application; however, for simplicity we have considered a single data gathering cycle. One cycle constitutes all sensor-nodes in a cluster sending one packet to the $\mathrm{CH}$, and $\mathrm{CH}$ sends it to a $\mathrm{ZM}$. When one cycle completes the $\mathrm{CH}$ would have received 19 packets from all member cluster sensor-nodes plus one packet from the $\mathrm{CH}$ node itself. Then the $\mathrm{CH}$ aggregates the data and sends it further to a ZM. Fig. 8 shows that LEACH and LEACH-C has high energy consumption with a slight difference, because both use the same mechanism for sending data to the basestation. This higher energy consumption is due to the fact that the CHs send the aggregated data directly to the far away base-station. While in BCDCP, the energy consumption is low, because it sends data through multi-hops to the basestation. However, ZBHF achieved lower energy consumption rate by employing local zone aggregator and hierarchical architecture. Furthermore, using ZM nodes in each zone (which are resource-rich heterogeneous nodes) takes the extra burden of sending aggregated packets to the base-station. In addition, the ZM is located in the middle of the zone, which is easily accessible by the $\mathrm{CH}$ in the zone without any extra cost. Therefore, the $\mathrm{CHs}$ avoid the long-haul communication. Furthermore, ZBHF allows only ZM nodes to communicate with the base-station so that the number of communications is decreased in the whole network.

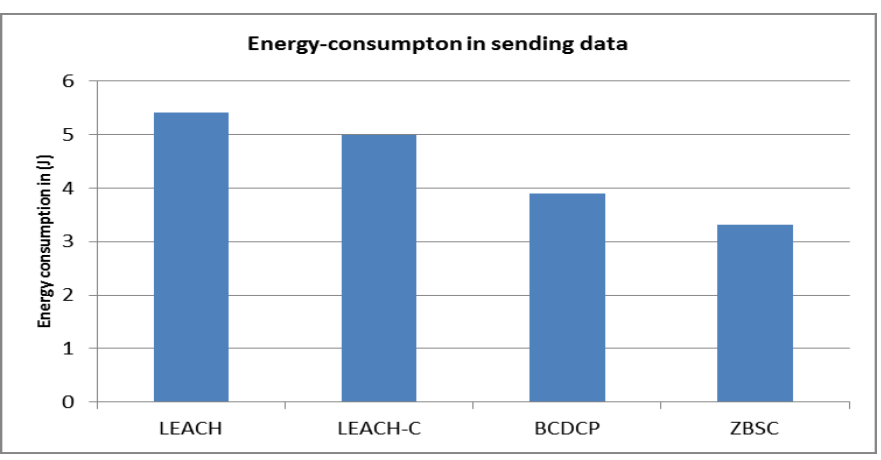

Fig.8. Case 3, The amount of energy consumed for sending sense data to the base-station in ZBHF

Case 4: In this case, we evaluate the change in the network lifetime with a different number of sensor-nodes, i.e. 80,160 and 320 . The number of $\mathrm{CHs}$ is $5 \%$, the same as for each of the network setups. According to the results shown in Fig. 9, the lifetime of $\mathrm{LEACH}$ and $\mathrm{LEACH}-\mathrm{C}$ is the same, while BCDCP and ZBSC have a slight difference for 80 sensornodes. However, the total lifetime in a network formed by 320 nodes in LEACH is much shorter. The reason is that the location information of nodes is not taken into account in the selection method of CHs in LEACH. Similarly, LEACH-C expends more energy in sending the data to the far away basestation, which exhausts its energy quickly. For 160 nodes, BCDCP and ZBSC have comparatively the same lifetime with only a slight difference; however, increasing the number of SNs in the network significantly reduces the network lifetime in $\mathrm{BCDCP}$ because the aggregated data at a given $\mathrm{CH}$ undergoes further processing as it hops along the $\mathrm{CH}$-to- $\mathrm{CH}$ routing. The proposed ZBSC showed an improved network lifetime due to the additional energy reduction effect through the zone-based hierarchical architecture and inception of ZM 
nodes, which transmit the aggregated data directly to the nearby ZM node.

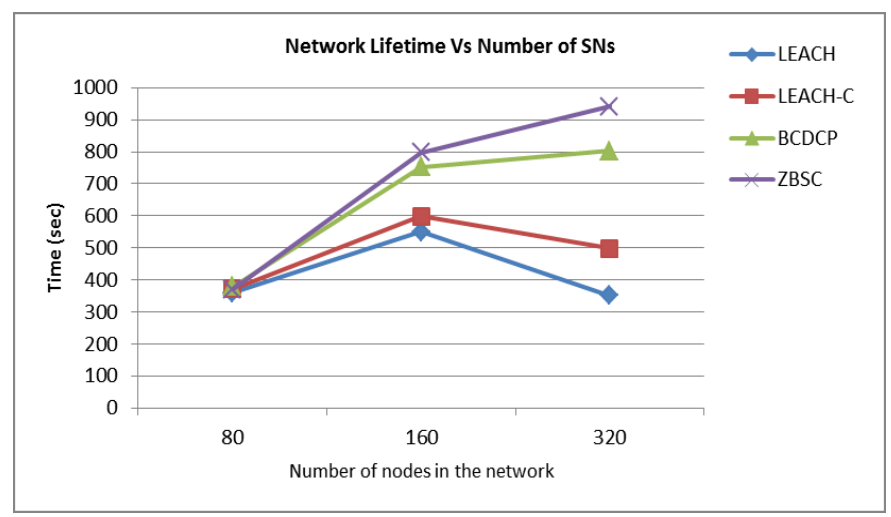

Fig.9. Case 4, Network lifetime

\section{CONCLUSION AND FUTURE WORK}

In this paper, we addressed the issue of energy-efficiency in WSNs in the context of network self-organization and clustering. We have proposed a novel and efficient network framework for an energy-efficient deployment of WSNs. We first proposed a new Zone-Based Hierarchical Framework (ZBHF) to deploy the network, followed by a new Zone-Based Self-Organization Clustering (ZBSC) scheme for energyefficient clustering. The main novelty in this work lies in proposing a new zone-based framework, which divides the network into $n$-zones and assigning each zone with a zonemanager node. Furthermore, the framework uses a $n$-tier hierarchal management approach of managing (e.g. $\mathrm{CH}$ and heterogeneous nodes) and managed node (e.g. SNs). The proposed ZBHF and ZBSC self-organize the network into clusters and significantly reduce the energy consumption during the network self-organization clustering process.

We evaluated the efficiency of the developed schemes through simulation experiments conducted in a customdesigned Java based simulator. The simulation results demonstrate that our proposed ZBSC scheme consumes less energy during the clustering process as compared to existing LEACH, LEACH-C and BCDCP schemes and maximize the network lifetime. In addition, a ZBHF provides a new network layout, which proved to consume less energy in sending sensed data to the base-station. Finally, we tested the proposed framework for various network sizes against change in the network lifetime. The simulation results verify that ZBHF and ZBSC provide efficient network infrastructure for maximizing network lifetime as compare to existing solutions. Relating to $\mathrm{ZBHF}$ and ZBSC as an ongoing work, we are investigating the issue of optimal placement location of a zone-manager in each zone. Furthermore, as part of our future work, we are committed to expand the proposed framework to incorporate some additional features of WSNs and to map it into a fault management architecture, which can provide efficient fault detection, diagnosis and recovery to make the network faulttolerant in the events of faults and failures.

\section{ACKNOWLEDGMENTS}

The author acknowledges University of Malakand (www.uom.edu.pk) and Higher Education Commission (HEC) of Pakistan for funding towards his research at Liverpool John Moores University, UK.

\section{REFERENCES}

[1] I. F. Akyildiz, W. Su, Y. Sankarasubramaniam, and E. Cayirci, "Wireless Sensor Networks: A Survey," Computer Networks, vol. 38, pp. 393-422, 2002.

[2] K. M. Sakib, "Energy Balanced Sensor Node Organization For Maximizing Network Lifetime," PhD, School of CS \& IT, RMIT University, Melbourne, Australia, 19 October, 2007.

[3] M. Z. Khan and I. M. Khan, "A Research Based Review of Wireless Sensor Networks," Annals. Computer Science Series, vol. 9, p. 16 , Dec. 2011.

[4] T. C. Collier and C. Taylor, "Self-organization in sensor networks," Jour. of Parallel and Distributed Computing, vol. 64, pp. 866-873, 2004.

[5] M. Kochhal, L. Schwiebert, and S. Gupta, "Role-based hierarchical self organization for wireless ad hoc sensor networks," in Proc. of the 2nd ACM Int. Conf. on Wireless Sensor Networks and Applications, 2003, pp. 98-107.

[6] A. A. Abbasi and M. Younis, "A survey on clustering algorithms for wireless sensor networks," in Computer Communications vol. 30, ed, 2007, pp. 2826-2841.

[7] M. Z. Khan, M. Merabti, B. Askwith, and F. Bouhafs, "A FaultTolerant Network Management Architecture for Wireless Sensor Networks," presented at the 11th Annual PGNet 2010 Conference, LJMU, Liverpool, UK, June 2010.

[8] V. Katiyar, N. Chand, and S. Soni, "A Survey on Clustering Algorithms for Heterogeneous Wireless Sensor Networks," Int. Jour. of Advanced Networking and Applications, vol. 02, pp. 745-754, 2011.

[9] G. Anastasi, M. Conti, M. Di Francesco, and A. Passarella, "Energy Conservation in Wireless Sensor Networks: A survey," $\mathrm{Ad}-\mathrm{Hoc}$ Networks, vol. 7, pp. 537-568, 2009.

[10] W. B. Heinzelman, A. P. Chandrakasan, and H. Balakrishnan, "An application-specific protocol architecture for wireless microsensor networks," IEEE Trans. on Wireless Communication, vol. 1, pp. 660$670,2002$.

[11] W. R. Heinzelman, A. Chandrakasan, and H. Balakrishnan, "EnergyEfficient Communication Protocol for Wireless Sensor Networks," presented at the Proc. of the 33th Hawaii Int. Conf. on System Sciences, 2000.

[12] S. D. Muruganathan, D. C. F. Ma, R. I. Bhasin, and A. O. Fapojuwo, "A centralized energy-efficient routing protocol for wireless sensor networks," IEEE Communications Magazine, vol. 43, pp. S8-13, 2005.

[13] S. Selvakennedy, S. Sinnappan, and Y. Shang, "A biologicallyinspired clustering protocol for wireless sensor networks," Computer Communications, vol. 30, pp. 2786-2801, 2007.

[14] N. Enami, R. A. Moghadam, K. Dadashtabar, and M. Hoseini, "Neural Network Based Energy Efficiency in Wireless Sensor Networks: A Survey," Int. Jour. of Comp. Sci. \& Eng. Survey (IJCSES), vol. 1, p. 17, August 2010.

[15] A. K. Gautam, "Accurate Localization Technique using Virtual Coordinate System in Wireless Sensor Networks," Int. Journal of Recent Trends in Engineering, vol. 2, 2009.

[16] K. Kifayat, M. Merabti, Q. Shi, et al., "An Efficient Multi-Parameter Group Leader Selection Scheme for Wireless Sensor Networks," in Proc. of the IFIP Int. Conf. on Network and Service Security, N2'09, Paris, France, June 2009.

[17] K. Kifayat, M. Merabti, Q. Shi, and D. Llewellyn-Jones, "An Efficient Multi-Parameter Group Leader Selection Scheme for Wireless Sensor Networks," presented at the 8th Annual PGNET'07 Conference, Liverpool, Uk, 2007. 